\title{
Hacia una Nueva Metodología de Diseño de Proyectos de Inversión Pública
}

David Medianero Burga

Resumen: El presente artículo contiene un avance de la propuesta metodológica elaborada por el suscrito en el marco de un trabajo de investigación que realiza en el Instituto de Investigaciones Económicas de la Facultad de Ciencias Económicas de la UNMSM, pero que se viene construyendo en permanente interacción con oficiales de proyectos del Gobierno Peruano, organismos de financiación de proyectos nacionales y diversos organismos de cooperación internacional. El objetivo es dotar al Gobierno Peruano de una metodología simplificada de diseño de proyectos de inversión pública que integre los métodos cualitativos y cuantitativos aplicados a los procesos de identificación, formulación y evaluación de proyectos. Esta metodología permite establecer la relación entre el enfoque del marco lógico y el flujo de caja de la alternativa implícitamente seleccionada como la óptima para la solución de un determinado problema. De este modo, se facilita el desarrollo de proyectos coherentes y realistas, unificando el lenguaje de formuladores y evaluadores $y$, por ende, rompiendo la tradicional barrera existente entre los especialistas en aspectos cualitativos y aquellos orientados a los aspectos cuantitativos en el análisis de proyectos. En buena cuenta, la metodología recomendada elevaría la calidad de las propuestas, al tiempo que disminuiría sustancialmente el costo de los procesos de preinversión.

Palabras clave: inversión pública, identificación, formulación, evaluación. 


\section{David Medianero Burga}

\section{Introducción}

La metodología contempla, en primer lugar, la identificación del problema central, pues, todos los proyectos inician su preparación cuando la población y/o las entidades ejecutoras son conscientes de la existencia de un problema. Acto seguido se procede a realizar el análisis de involucrados, buscando delimitar la población que potencialmente será la directamente beneficiaria. En tercer lugar, se realiza el análisis de problemas, haciendo uso de la metáfora gráfica del árbol de problemas. Este se trueca en su inversa, el árbol de objetivos, lo que facilita el análisis de objetivos y alternativas, que constituye el cuarto paso. Luego de seleccionada la alternativa, en base a la cuantificación de criterios estrictamente cualitativos, se deriva el marco lógico, quinto paso y eslabón entre los instrumentos cualitativos y cuantitativos. A partir de aquí se desarrollan, en los pasos del seis al diez, una serie de estimaciones cuantitativas que concluyen en la determinación del plan de implementación, los costos y beneficios del proyecto, la evaluación a través del flujo de caja y, finalmente, la matriz de monitoreo y evaluación. Esta secuencia permite el cálculo de los indicadores de rentabilidad social, que es la base para la decisión final del Estado: aceptar, postergar o rechazar el proyecto.

De esta manera, los métodos cuantitativos y cualitativos de preparación de proyectos son integrados en un cuerpo metodológico único que, partiendo de la identificación del problema central, culmina en el cálculo de la rentabilidad social de la inversión. Los dos instrumentos más importantes del estado del arte en economía y finanzas públicas en lo que se refiere a preparación de proyectos -el marco lógico y el flujo de caja- son reunidos en un procedimiento de diez pasos que permite diseñar proyectos coherentes, realistas y evaluables. La metodología se sustenta en los fundamentos del análisis de proyectos, en calidad de intervenciones estratégicas del Estado en el proceso de desarrollo. La metodología permite desarrollar paso-a-paso la construcción de un proyecto. Como resultará de esperar, ella conduce a la obtención de un VAN ${ }^{1}$ social y otros indicadores de rentabilidad social, pero a diferencia de lo que se obtiene en los textos de evaluación de proyectos (y lamentablemente lo que se hace en la totalidad de proyectos sociales) esta vez se mantiene a la vista la relación entre el problema social a solucionar, la población beneficiaria potencial y los distintos elementos del marco lógico del proyecto. 


\section{Hacia una nueva metodología de diseño de proyectos de inversión pública}

Proyecto : Museo del Queso Cajamarquina, Centro de Arte y Tradicion Quesera

Formato : Análisis de involucrados

\begin{tabular}{|c|c|c|c|c|c|}
\hline Actores / involucrados & Nímero & Actitudes & Recursos & $\begin{array}{l}\text { Importancia } \\
\text { para el exito } \\
\text { del proyecto }\end{array}$ & $\begin{array}{l}\text { Grado de } \\
\text { infuancia sobre } \\
\text { el proyecto }\end{array}$ \\
\hline $\begin{array}{l}\text { PYMES de transtomación de lácteos de las provincias de } \\
\text { Cajamarca, Hualgayoc , San Miguel y Celendin }\end{array}$ & 116 & 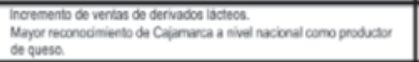 & $\begin{array}{l}\text { Disponiblidad de producción de derivados lacteos } \\
\text { Mano de dora no colficada. } \\
\text { Herramientas. }\end{array}$ & 5 & 3 \\
\hline $\begin{array}{l}\text { Asociaciones de productores de derivadcs ládeos de las } \\
\text { provincias de Cajamarca Hualgayoc y Tongod. }\end{array}$ & 3 & 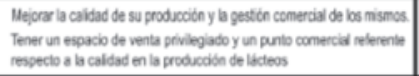 & $\begin{array}{l}\text { Trabajo y conocimiento previo de las PYMES de } \\
\text { transilormadón de liscleos }\end{array}$ & 4 & 5 \\
\hline Empresas u cperadores rebcionados al sector turismo. & P.D. & Mayor pemanencia de los turistas en Cajamarca. & $\begin{array}{l}\text { Infraestructura (hoteles, restaurantes, etc) } \\
\text { Conocimiento de la cadena de senvicios turisticos }\end{array}$ & 4 & 3 \\
\hline Municipalidad Provincial de Cajamarca & 1 & Lograr el impacto deseado en empleo, ingresos y desarrollo. & Humanos, Financieros, Materiales. & 4 & 4 \\
\hline Servicio Holandés de Cooperación al Desarrollo & 1 & Lograt ef impacto deseado en empleo, ingresos y desartollo. & Humanos, Financieros, Materiales. & 4 & 4 \\
\hline Centro Eoundérico de Promoción y Acción Social Norte & 1 & Lograr el impacto deseado en empleo, ingresos y desarolo. & Humanos, Financieros, Materiales. & 4 & 4 \\
\hline CTN & 1 & Lograr el impacto deseado en empleo, ingresos y desartolo. & Humanos, Finandieros, Materiales. & 4 & 4 \\
\hline $\begin{array}{l}\text { Productores de leche y quesillo de la provincia de } \\
\text { Cajamarca. }\end{array}$ & P.D. & Incremento de la demanda de leche como materia prima. & $\begin{array}{l}\text { Disponibilidad de producción de leche y quesilo } \\
\text { Mano de obra no calificada. }\end{array}$ & 3 & 3 \\
\hline $\begin{array}{l}\text { Coordinadora de Desamollo del Sector de Denivados } \\
\text { Lácteos (CookLAC) }\end{array}$ & 1 & Mejorar las condiciones de vida de los productiones isctieos & Humanos, Financieros, Materiales. & 2 & 2 \\
\hline Universidad (UNC) & 1 & Promover of desarrolo econdimicos productivo de Cajamarca y al pais & Humanos, Materialos. & 2 & 2 \\
\hline $\begin{array}{l}\text { Laboratorio Regional del Nonte de Sanicad Animal de } \\
\text { Cajamarca (LABRENOR) }\end{array}$ & 1 & 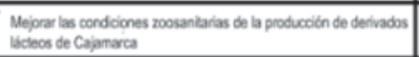 & Humanos, Materales. & 2 & 2 \\
\hline Tunstas nacionales & 17,520 & 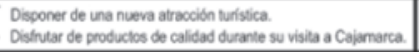 & Financieros & 1 & 1 \\
\hline Tunistas extranjeros & 26,280 & $\begin{array}{l}\text { Disponer de una nueva atracolion turistica } \\
\text { Distruar de produclos de calidad durante su vista a Cajamarca. }\end{array}$ & Financieros & 1 & 1 \\
\hline
\end{tabular}

(*) Se veliza una escala de 1 a 5 , calificando de 1 al de menor importancia e infuencia y 5 al de mayor importancia e influencia.

Como es sabido, en el contexto de la promoción del desarrollo, un proyecto es un conjunto de actividades interrelacionadas destinadas a lograr un objetivo específico de desarrollo, a un costo y tiempo predeterminados. Un proyecto social tiene como objetivo la solución de un problema social, ya sea que éste consista en una necesidad básica insatisfecha o en una oportunidad de mercado desaprovechada. Por otra parte, un proyecto implica la utilización de recursos y su transformación en productos o resultados a través de la realización de determinadas actividades. Un proyecto, cualquiera sea su naturaleza, representa el interés de la sociedad por ofrecer una solución a un problema social, e implica un intercambio entre costos y beneficios. Toda inversión exige la reducción de un beneficio en el presente con la esperanza de recibir algún beneficio en el futuro.

Bajo estas amplias consideraciones conceptuales, se ha buscado combinar las técnicas cuantitativas y cualitativas integrándolas en un cuerpo metodológico único que permite abordar los procesos de identificación, formulación y evaluación de proyectos, operativizándolos en los diez pasos siguientes: 


\section{David Medianero Burga}

Paso 1. Determinación del problema central.

Paso 2. Análisis de involucrados.

Paso 3. Análisis de problemas.

Paso 4. Análisis de objetivos y alternativas.

Paso 5. Elaboración del marco lógico.

Paso 6. Elaboración del plan de implementación.

Paso 7. Estimación de costos.

Paso 8. Estimación de beneficios.

Paso 9. Evaluación del proyecto.

Paso 10. Sistema de monitoreo y evaluación.

La metodología permite, además, trabajar las relaciones lógicas y numéricas con la ayuda de las hojas de cálculo y luego, con base en los datos y la información contenida y obtenida en dichas tablas, redactar el documento de proyecto en base a un formato que, por su parte, permite comunicar adecuadamente la naturaleza de la intervención, así como su viabilidad, impacto y sostenibilidad.

\section{Determinación del problema central}

La selección del problema que el proyecto intentará solucionar es el paso y la decisión más importante del proceso de preinversión. Los proyectos sociales son, esencialmente, grandes respuestas a grandes problemas. Por ello mismo, el error más grave que se puede cometer en diseño de proyectos consiste en intentar resolver con precisión el problema equivocado.

Para la determinación del problema central, se propone la utilización del instrumento metodológico denominado Matriz de Selección del Problema Central. Este permite comparar los problemas identificados en función de criterios clave para la implementación de un proyecto, tales como: pertinencia, viabilidad y nivel de incidencia. 
Hacia una nueva metodología de diseño de proyectos de inversión pública

En el ejemplo adjunto, la Matriz de Selección del Problema Central ha permitido priorizar el segundo problema, referido a la débil promoción de los sectores lácteos y el turismo en Cajamarca.

Proyecto: Museo del Queso Cajamarquino,
Centro de Arte y Tradición Quesera
\begin{tabular}{|l|c|c|c|c|c|c|}
\hline \multicolumn{1}{|c|}{ Problemas } & 1 & 2 & 3 & 4 & 5 & $\begin{array}{c}\text { Totales } \\
\text { horizontales }\end{array}$ \\
\hline 1. Bajos ingresos del sector de servicios & & 1 & 0 & 0 & 0 & 1 \\
\hline $\begin{array}{l}\text { 2. Débil promoción de los sectores lácteos y } \\
\text { turismo en Cajamarca }\end{array}$ & 0 & & 0 & 0 & 0 & 0 \\
\hline $\begin{array}{l}\text { 3. Limitados medios de comunicación a } \\
\text { Cajamarca }\end{array}$ & 1 & 1 & & 1 & 1 & 4 \\
\hline $\begin{array}{l}\text { 4. Mano de obra de la localidad } \\
\text { desempleada }\end{array}$ & 1 & 1 & 0 & & 1 & 3 \\
\hline $\begin{array}{l}\text { 5. Escaso diálogo y coordinación entre } \\
\text { sectores productivos }\end{array}$ & 1 & 1 & 0 & 0 & & \\
\hline & 3 & 4 & 0 & 1 & 2 & 2 \\
\hline
\end{tabular}

\section{Análisis involucrados}

El análisis de involucrados consiste en identificar a los grupos que se relacionan directa o indirectamente con la problemática bajo análisis, lo cual permitirá a la postre desarrollar acciones dirigidas a optimizar los beneficios sociales e institucionales del proyecto y limitar los impactos negativos. Identificar a los involucrados significa conocer qué actores se movilizarán con relación al proyecto, teniendo en cuenta no solo su posición actual, sino también su posición futura. Un elemento fundamental en el análisis de involucrados es la determinación de la población beneficiaria. Esto no sólo permite orientar el diseño de la intervención en lo que se refiere, por ejemplo, al análisis de alternativas, sino también en la elaboración del flujo de caja y el cálculo subsiguiente de los indicadores de rentabilidad. Debe recordarse que el VAN de un proyecto social constituye, esencialmente, la utilidad que el proyecto generará para la población beneficiaria.

Sobre la base de la identificación de los agentes involucrados, se establece una matriz en la cual se señalan en una breve síntesis los grados de interés, recursos, así como la importancia y grado de influencia que tienen respecto al éxito del proyecto cada uno de los agentes. 


\section{David Medianero Burga}

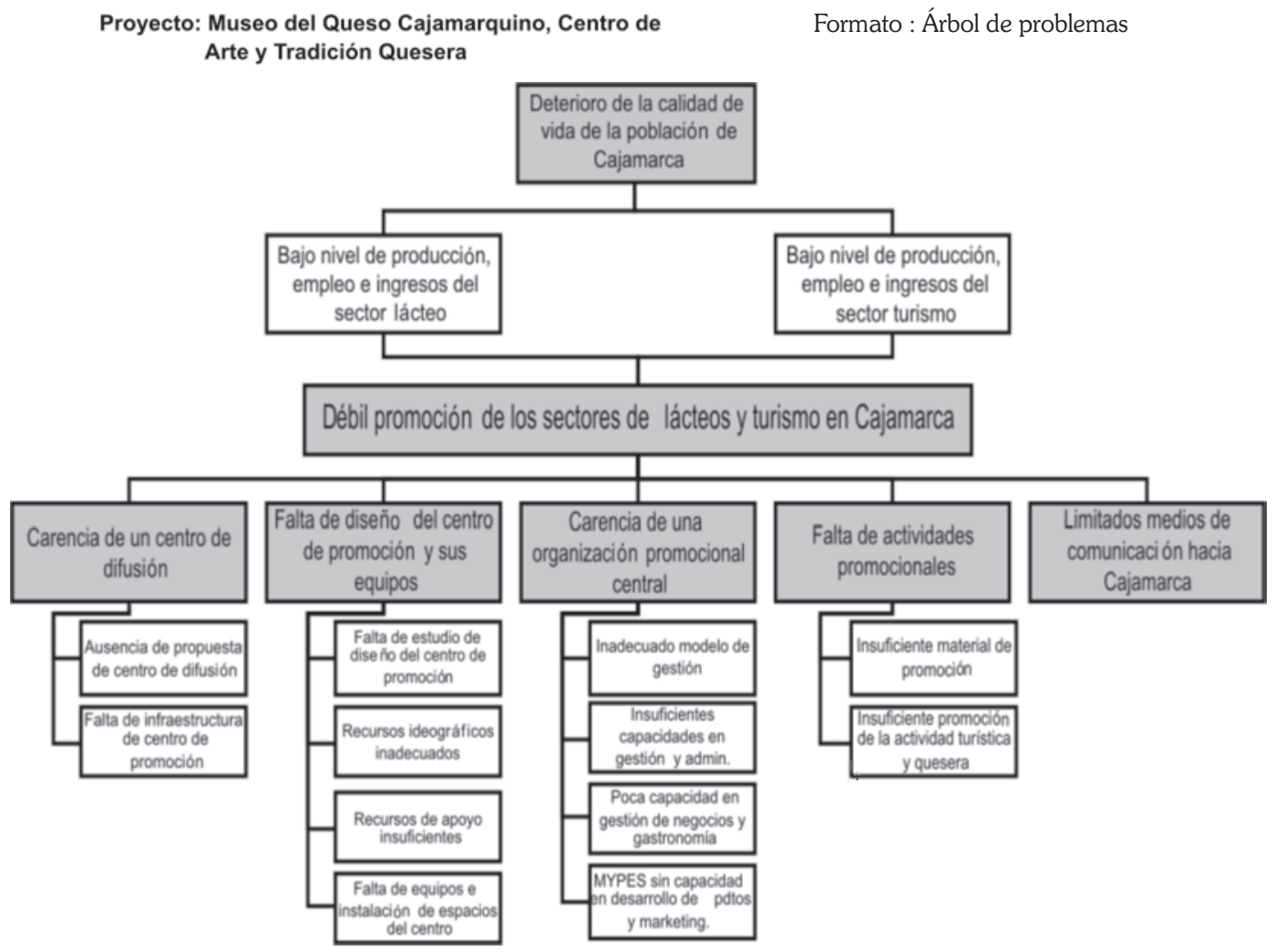

En la Matriz de Análisis de Involucrados adjunta se muestra en detalle los actores relevantes del proyecto del Museo del Queso Cajamarquino.

\section{Análisis de problemas}

El análisis de problemas tiene como propósito fundamental la correcta determinación de las causas que originan un problema previamente identificado, en el entendimiento de que el conocimiento de las causas sirve como pauta para la determinación de las alternativas de solución. Si bien el análisis del problema central se efectúa en términos cualitativos, en las etapas avanzadas de diseño del proyecto puede ser efectuado en forma cuantitativa, dando como resultado la construcción de la línea de base del proyecto. El 
Hacia una nueva metodología de diseño de proyectos de inversión pública

análisis de problemas permite determinar aquellos factores que tienen incidencia en el problema. Para solucionar un problema es requisito indispensable entenderlo correctamente, lo cual implica la identificación y comprensión de sus causas más relevantes.

Una vez que ha sido determinado el problema fundamental, se analizan sus causas y sus efectos. El punto de partida para solucionar un problema es analizarlo correctamente, con la ayuda de un instrumento metodológico denominado "Arbol de problemas". El análisis de efectos consiste en representar gráficamente los efectos surgidos a consecuencia del problema.

El árbol de problemas de este caso muestra las cinco causas fundamentales que inciden en la débil promoción de los sectores de lácteos y turismo en Cajamarca.

\section{Análisis de objetivos y alternativas}

El análisis de objetivos consiste en identificar las posibles soluciones a un problema identificado. Se realiza a través de la conversión de las expresiones señaladas en el árbol de problemas, en situaciones positivas, formulando así el árbol de objetivos. El árbol de objetivos muestra los distintos medios disponibles para la solución del problema. En este paso, el problema principal se convierte en objetivo principal, las causas en medios y, finalmente, los efectos en fines. La realización del análisis de objetivos y alternativas permite a la entidad formuladora del proyecto identificar los medios posibles para la solución del problema y seleccionar aquellos que resulten los más adecuados para su solución. Una vez identificados los medios, se puede proceder en forma concreta al diseño del proyecto.

El análisis de objetivos se realiza mediante el uso del instrumento denominado árbol de objetivos, que es una versión en positivo del árbol de problemas, donde los medios fundamentales se especifican en el nivel inferior y constituyen las raíces del árbol. Los fines por su parte, se especifican en la parte superior.

En el siguiente ejemplo, el Árbol de Objetivos señala que el proyecto se orienta a la consolidación de los sectores de lácteos y turismo en Cajamarca a través de la construcción del Museo del Queso Cajamarquino. 


\section{David Medianero Burga}

\section{Proyecto: Museo del Queso Cajamarquino, Centro de Arte y Tradición Quesera

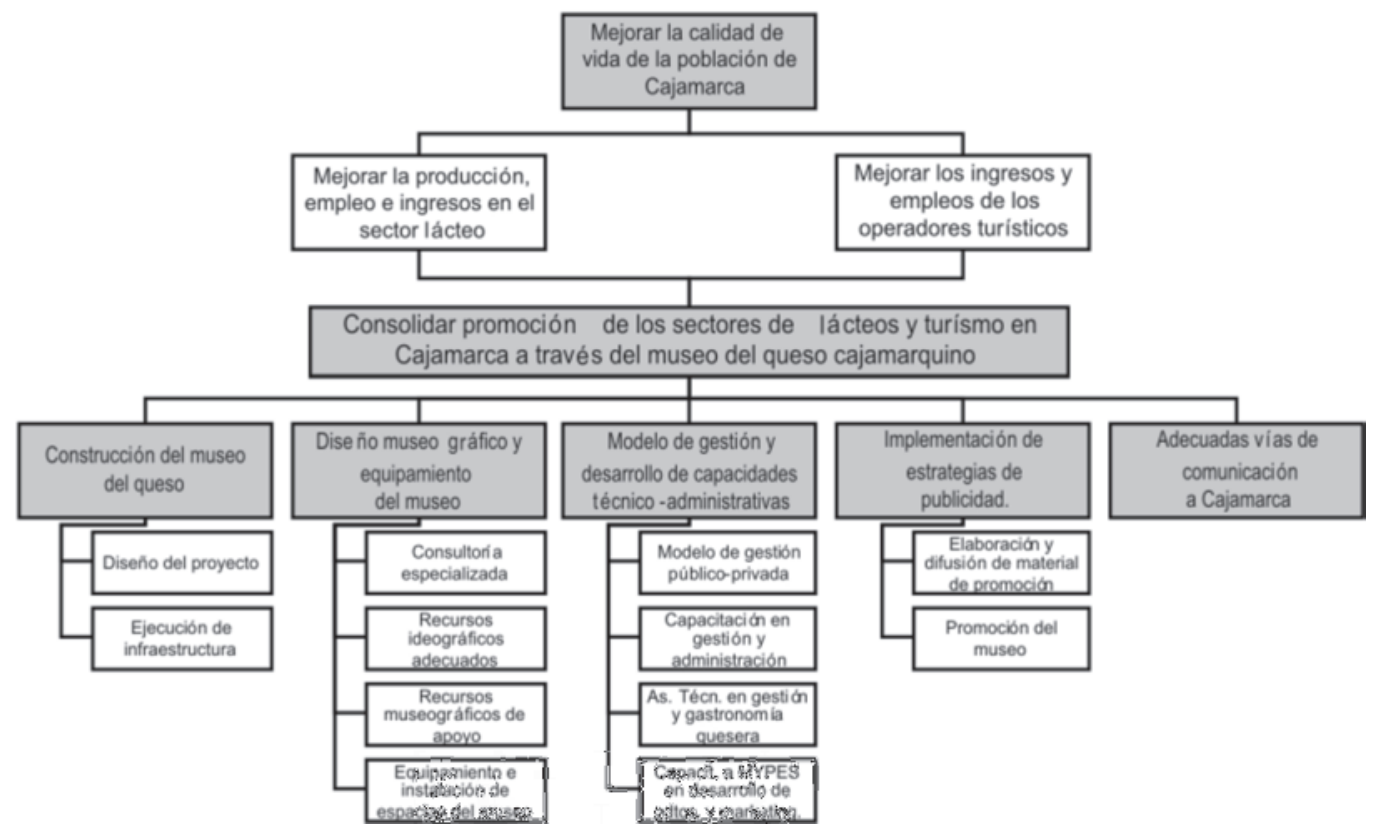

\section{Marco lógico}

El marco lógico es, en esencia, una matriz conceptual para organizar los distintos elementos de un proyecto. Permite un diseño que satisface tres requerimientos fundamentales de calidad en los proyectos: coherencia, viabilidad y evaluabilidad. La matriz del marco lógico constituye el documento maestro del proyecto, del cual se puede obtener en forma rápida el plan de acción del proyecto, los recursos necesarios para su ejecución y la lógica sobre la cual descansa el proyecto. Permite realizar comparaciones con celeridad y efectuar las correcciones que se consideren pertinentes. El marco lógico es la "maqueta" de un proyecto de desarrollo: brinda no sólo los elementos esenciales de la intervención, sino que también permite visualizarlos.

Con la elaboración del marco lógico, la etapa de identificación del proyecto ha concluido, pudiéndose expresar documentariamente a través de un perfil de proyecto. En los pasos siguientes, conforme se va desarrollando la matriz del marco lógico, se irá adicionando nueva información en los distintos casilleros o corrigiendo la ya existente, lo cual se vera reflejado, 


\section{Hacia una nueva metodología de diseño de proyectos de inversión pública}

asimismo, en nuevos documentos del proyectos, tales como nuevas versiones del perfil, propuestas, estudios de factibilidad u otros.

Proyecto: Museo del Queso Cajamarquino, Centro de Arte y Tradición Quesera

Formato : Matriz del marco lógico

\begin{tabular}{|c|c|c|c|}
\hline OBJETIVOS & INDICADORES & MEDIOS DE VERIFICACION & SUPUESTOS \\
\hline \multicolumn{4}{|l|}{ FIN } \\
\hline $\begin{array}{l}\text { Contribuir al incremento de los ingresos de los } \\
\text { productores de derivados lácteos artesanales y } \\
\text { de las empresas relacionadas con el sector } \\
\text { turismo. (Operadores turisticos) }\end{array}$ & $\begin{array}{l}\text { Ingresos por ventas de las MYPES de lácteos. } \\
\text { Ingresos por ventas de los operadores turisticos. } \\
\text { Número de empleos directos generados }\end{array}$ & $\begin{array}{l}\text { Encuestas a PYMES al concluir el proyecto. } \\
\text { INEIENAHO }\end{array}$ & Estabilidad Macroeconómica y social. \\
\hline \multicolumn{4}{|l|}{ PROPOSITO } \\
\hline $\begin{array}{l}\text { Implementar y hacer funcionar el museo del } \\
\text { queso Cajamarquino como una nueva actividad } \\
\text { turistica en el Circuito Regional Norte. }\end{array}$ & $\begin{array}{l}\text { Número de visitantes del museo. } \\
\text { Número de productores ylo empresas } \\
\text { desarrollando mejores tecnologias. } \\
\text { Tiempo promedio de permanencia del turista en } \\
\text { Cajamarca. }\end{array}$ & $\begin{array}{l}\text { Registro de boleteria del museolibros contables. } \\
\text { Actas de constitución de empresas. } \\
\text { Encuesta a agencias y operadores turisticos. }\end{array}$ & $\begin{array}{l}\text { Flujo estable de crecimiento turistico en } \\
\text { Cajamarca. }\end{array}$ \\
\hline \multicolumn{4}{|l|}{ COMPONENTES } \\
\hline 1. Diseño y construcción de la infraestructura. & Area de infraestructura construida. & $\begin{array}{l}\text { Verificación directa. } \\
\text { Archivo fotografico del museo }\end{array}$ & Disponiblilidad de recursos en forma oportuna. \\
\hline II. Diseño museográfico y equipamiento & $\begin{array}{l}\text { Número de colecciones. } \\
\text { Número de equipos }\end{array}$ & $\begin{array}{l}\text { Documentos de informes de consultorias. } \\
\text { Verificación directa. }\end{array}$ & Disponibilidad de recursos en forma oportuna. \\
\hline $\begin{array}{l}\text { III. Modelo de gestión y desarrollo de capacidades } \\
\text { técnico-administrativas }\end{array}$ & $\begin{array}{l}\text { Museo constituido legalmente } \\
\text { Número de trabajadores capacitados }\end{array}$ & $\begin{array}{l}\text { Estatutos de empresa. } \\
\text { Registro de capacitación }\end{array}$ & $\begin{array}{l}\text { Permanencia de las personas a cargo de la } \\
\text { administración del museo. } \\
\text { Existencia de interés permanente de los } \\
\text { participantes. }\end{array}$ \\
\hline IV. Implementación de estrategias de publicidad. & $\begin{array}{l}\text { Número de operadores turisticos que incorporan } \\
\text { al museo como parte de su oferta turistica. }\end{array}$ & Guias Turisticas regionales & $\begin{array}{l}\text { Los compromisos de las instituciones vinculada } \\
\text { al sector turismo se cumpien }\end{array}$ \\
\hline V. Se ha gestionado eficazmente el proyecto. & Número de informes de monitoreo y evaluación. & Reportes & \\
\hline \multicolumn{4}{|l|}{ ACTIVIDADES } \\
\hline Varias por cada componente & $\begin{array}{l}\text { COSTOS } \\
\text { Total SI. } \\
\text { ALAC } \\
\text { LWR } \\
\text { CEDEPAS } \\
\text { MPC } \\
\text { SNV } \\
\text { CTN } \\
\text { OTROS } \\
\end{array}$ & $\begin{array}{l}\text { Estados financieros y presupuestarios del } \\
\text { proyecto. }\end{array}$ & Participación de los beneficiarios \\
\hline
\end{tabular}

\section{Plan de implementación}

El plan de implementación, plan operativo o programación de metas físicas y financieras, es un instrumento que organiza en forma simultánea la información sobre las actividades y los gastos. Contiene el cronograma de las actividades (metas físicas) y el cronograma de gastos (metas financieras). Constituye el principal instrumento de gerencia de proyectos y, a su vez, la referencia fundamental para las acciones de monitoreo, razón por la cual debe ser realizado de la manera más realista y participativa posible. Tomando como base el plan de implementación, se elaboran los informes técnico-financieros, a través de los cuales se informa sobre el avance en la ejecución del proyecto. En conjunto, el plan de implementación y los informes técnicofinancieros, ayudan a la toma de decisiones operativas durante la ejecución del proyecto.

La programación de metas físicas y financieras establecidas en el presente caso muestra un horizonte de ejecución de dos años, proyectándose realizar la mayor parte de los desembolsos al primer año. El formato respectivo para el caso del Museo del Queso Cajamarquino se muestra a continuación: 
David Medianero Burga

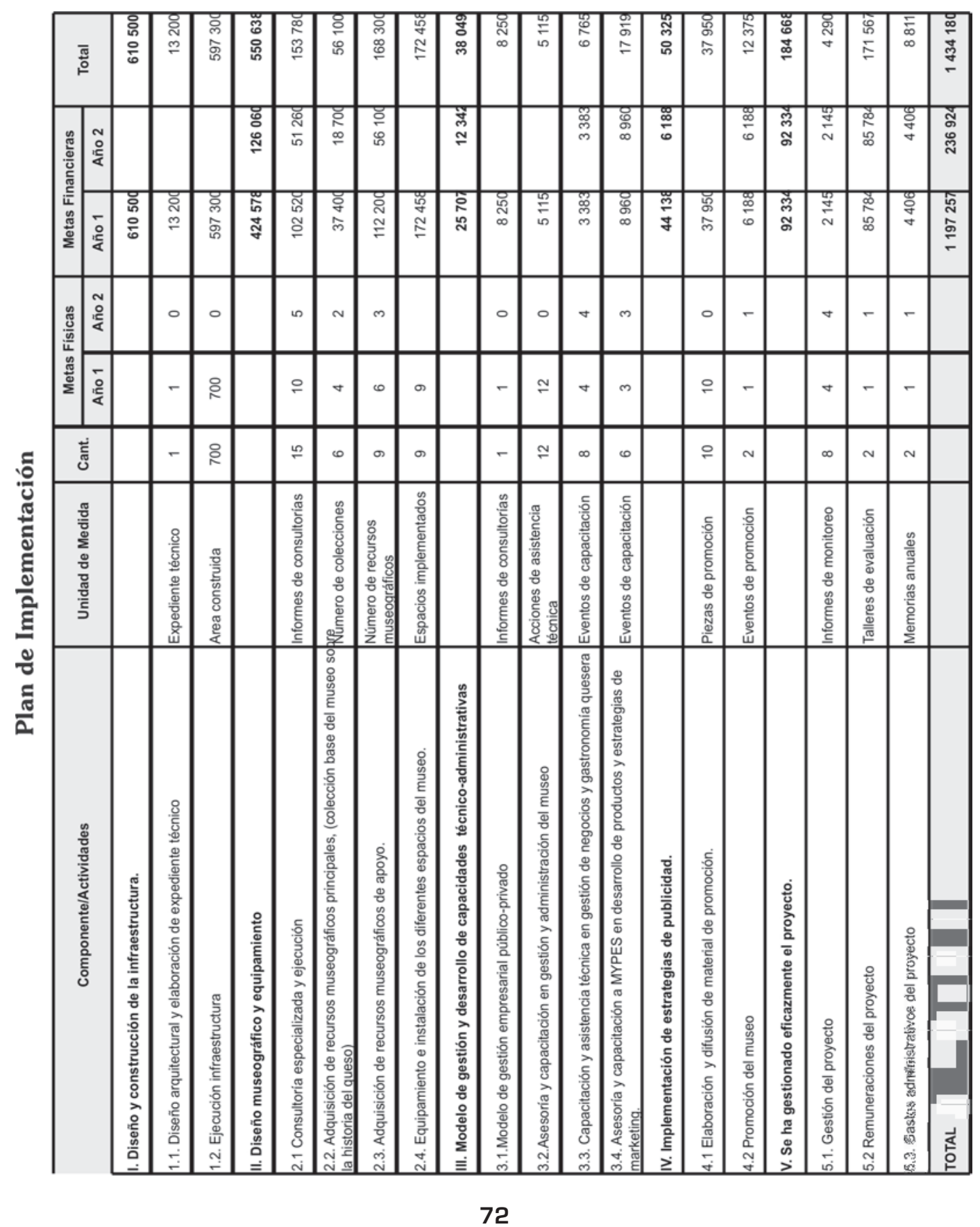


Hacia una nueva metodología de diseño de proyectos de inversión pública

\section{Costos del proyecto}

El análisis de los costos de un proyecto consiste en la determinación de los recursos económicos necesarios para la ejecución de las actividades previstas en el plan operativo global. La ejecución del proyecto supondrá dos tipos de costos: costos de inversión y costos de operación. Los costos de inversión están vinculados al proceso inicial de implementación del proyecto y se corresponden con la ejecución de las actividades planificadas en el marco lógico. Los costos de operación, por su parte, son los que se generan durante todo el periodo en el que el proyecto se encuentra en funcionamiento. Para fines de evaluación, los costos operativos relevantes son los costos operativos incrementales, que resultan de la diferencia entre los costos operativos que se generan en la situación con proyecto menos los costos operativos que se proyectan si la intervención no fuera implementada.

El presupuesto de un proyecto puede elaborarse atendiendo a dos enfoques de costeo diferentes pero complementarios: (i) costeo por actividades/componentes, en cuyo caso el presupuesto se determina a partir del costeo de cada una de las actividades del proyecto; (ii) costeo por categorías de gasto, en cuyo caso el presupuesto se elabora a partir de la identificación de los recursos necesarios, agrupándolos en bloques o categorías, tales como sueldos, beneficios sociales, bienes, equipos, viáticos, etc.

Los costos de inversión y operativos incrementales del proyecto se muestran en detalle en el cuadro del presupuesto analítico que se presenta a continuación:

\begin{tabular}{|l|l|l|r|r|}
\hline $\begin{array}{l}\text { III. Modelo de gestión y desarrollo de } \\
\text { capacidades tecnico-administrativas }\end{array}$ & & & & 38049 \\
\hline $\begin{array}{l}\text { 3.1.Modelo de gestión empresarial público- } \\
\text { privado }\end{array}$ & & & & 8250 \\
\hline Consultoría & Global & 1 & 8250 & 8250 \\
\hline $\begin{array}{l}\text { 3.2.Asesoría y capacitación en gestión y } \\
\text { administración del museo }\end{array}$ & & & & 5115 \\
\hline 3.2.1 Capacitación & & & & $\mathbf{5 1 1 5}$ \\
\hline
\end{tabular}




\begin{tabular}{|l|r|r|r|r|}
\hline Material de capacitación & Global & 1 & 1815 & 1815 \\
\hline Capacitador & cursos & 4 & 825 & 3300 \\
\hline $\begin{array}{l}\text { 3.3. Capacitación y asistencia técnica en } \\
\text { gestión de negocios y gastronomia quesera }\end{array}$ & & & & 6765 \\
\hline 3.3.1.Capacitación & & & & 6765 \\
\hline Material de capacitación & Global & 1 & 1815 & 1815 \\
\hline Capacitador & cursos & 6 & 825 & 4950 \\
\hline $\begin{array}{l}\text { 3.4. Asesoría y capacitación a MYPES en } \\
\text { desarrollo de productos y estrategias de } \\
\text { marketing. }\end{array}$ & & & & 17919 \\
\hline Material de capacitación & & & & \\
\hline Capacitación & Global & 1 & 3399 & 3399 \\
\hline $\begin{array}{l}\text { IV. Se ha implementado estrategias eficaces de } \\
\text { publicidad }\end{array}$ & Eventos & 4 & 3630 & 14520 \\
\hline $\begin{array}{l}\text { 4.1.Elaboración y difusión de material de } \\
\text { promoción }\end{array}$ & & & & 50325 \\
\hline $\begin{array}{l}\text { 4.1.1 Diseño e impresión de materiales de } \\
\text { promoción }\end{array}$ & global & 1 & 26400 & 26400 \\
\hline 4.1.3 Difusión (Publicidad radial y televisiva) & global & 1 & 11550 & 11550 \\
\hline 4.2 Eventos de promoción & eventos & 3 & 4125 & 12375 \\
\hline V. Se ha gestionado eficazmente el proyecto & & & & 184668 \\
\hline 5.1 Gestión del Proyecto & & & & 4290 \\
\hline 5.1.1 Monitoreo & Global & 1 & 2970 & 2970 \\
\hline 5.1.2 Evaluación & Talleres & 4 & 330 & 1320 \\
\hline 5.2 Remuneraciones del Proyecto & & & & 171567 \\
\hline 5.2.1 Asesor del proyecto CEDEPAS & Mensual & 12 & 990 & 11880 \\
\hline 5.2.2 Asesor del proyecto SNV & 48 & 1320 & 63360 \\
\hline 5.2.3 Coordinador de Proyecto & Anual & 1 & 20790 & 20790 \\
\hline 5.2.4 Asistente contable & & & & 8811 \\
\hline 5.3 Gastos administrativos del Proyecto & & & & 1434180 \\
\hline APORTE TOTAL (SI.) & & & & 37950 \\
\hline
\end{tabular}


Hacia una nueva metodología de diseño de proyectos de inversión pública

Proyecto: Museo del Queso

Cajamarquino, Centro de Arte y

Tradición Quesera
Formato:

Presupuesto analítico

\begin{tabular}{|c|c|c|c|c|}
\hline \multirow{2}{*}{ COMPONENTE/ACTIVIDADES } & \multicolumn{2}{|c|}{ META } & \multirow{2}{*}{$\begin{array}{l}\text { COSTO } \\
\text { UNIT. }\end{array}$} & \multirow{2}{*}{$\begin{array}{l}\text { TOTAL } \\
\text { PPTO }\end{array}$} \\
\hline & UNID MED & CANT. & & \\
\hline I. Diseño y Construcción de la infraestructura & & & & 610500 \\
\hline 1.1. Diseño de Proyecto & & & 13200 & 13200 \\
\hline $\begin{array}{l}\text { 1.1.1. Elaboración de Expediente Técnico y diseño } \\
\text { arquitectonico }\end{array}$ & Honorarios & 1 & 13200 & 13200 \\
\hline 1.2. Ejecución de Infraestructura & & & & 597300 \\
\hline 1.1.1. Terreno $(1200 \mathrm{~m} 2)$ & $\mathrm{m} 2$ & 1,200 & 275 & 330000 \\
\hline $\begin{array}{l}\text { 1.1.2. Obras Civiles (sistema constructivo de tierra } \\
\text { y concreto) }\end{array}$ & global & 1 & 231000 & 231000 \\
\hline 1.1.3. Residencia de obra & Honorarios & 5 & 3300 & 16500 \\
\hline 1.1.4. Supervisión de Obras & Honorarios & 5 & 3960 & 19800 \\
\hline II. Diseño museográfico y equipamiento & & & 0 & 550638 \\
\hline 2.1 Consultorias especializada y ejecución & & & & 153780 \\
\hline $\begin{array}{l}\text { 2.2 Adquisición de recursos museográficos } \\
\text { principales. }\end{array}$ & & & & 56100 \\
\hline $\begin{array}{l}\text { 2.2.1 Recurso museográfico principal: colección } \\
\text { base del museo }\end{array}$ & & & & 56100 \\
\hline 2.2.2 Equipos y herramientas antiguos & Estm. & 1 & 33000 & 33000 \\
\hline 2.2.3 Equipos y herramientas modernos & Estm. & 1 & 23100 & 23100 \\
\hline $\begin{array}{l}2.3 \text { Adquisición de recursos museográficos de } \\
\text { apoyo: }\end{array}$ & & & & 168300 \\
\hline 2.4 Equipamiento e instalación de los espacios & & & & 172458 \\
\hline 2.4.1 Espacio Multimedia & & & & 28215 \\
\hline 2.4.2 Espacio Galeria de Arte & & & & 35607 \\
\hline 2.4.3 Espacio Educativo y Biblioteca & & & & 42900 \\
\hline 2.4.4 Administración y servicios & & & & 34485 \\
\hline Muebles de oficina, computadoras, fax & Global & 1 & 33000 & 33000 \\
\hline Diseño pagina webb & Honorarios & 1 & 1485 & 1485 \\
\hline 2.4.5 Espacio de Promoción y Ventas & & & & 18051 \\
\hline Equipamiento & Global & 1 & 18051 & 18051 \\
\hline 2.4.6 Corrales para Ganado & & & & 13200 \\
\hline Compra de animales (5 vacas ) & Estm. & 5 & 2640 & 13200 \\
\hline
\end{tabular}


David Medianero Burga

\begin{tabular}{|c|c|c|c|c|c|c|c|c|c|c|c|c|c|c|c|c|c|c|}
\hline 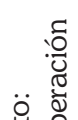 & 웅 & $\begin{array}{l}\frac{10}{50} \\
\frac{10}{2}\end{array}$ & $\begin{array}{l}\bar{\emptyset} \\
\text { 흔 }\end{array}$ & $\begin{array}{l}\frac{O}{N} \\
\text { J़ }\end{array}$ & $\frac{\widehat{\infty}}{\oplus}$ & $\frac{\Xi}{\frac{\Xi}{9}}$ & $\stackrel{\frac{\pi}{\omega}}{\underline{\omega}}$ & 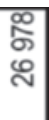 & $\begin{array}{l}\text { 岕 } \\
\text { o }\end{array}$ & $\begin{array}{l}\text { జ్ర } \\
\sim\end{array}$ & $\begin{array}{l}8 \\
8 \\
ㅇ ㅡ\end{array}$ & $\begin{array}{l}8 \\
8 \\
\text { 이 }\end{array}$ & $\underset{0}{\mathscr{ల}}$ & $\stackrel{\substack{\text { ले } \\
\sim}}{ }$ & $\begin{array}{l}\text { প్ల } \\
\text { m }\end{array}$ & 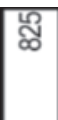 & 0 & 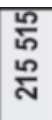 \\
\hline 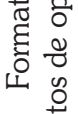 & $\begin{array}{l}0 \\
\text { \&े }\end{array}$ & $\begin{array}{l}\text { 尔 } \\
\frac{1}{2}\end{array}$ & 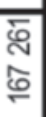 & $\begin{array}{l}\frac{9}{N} \\
\text { J }\end{array}$ & $\stackrel{\frac{\pi}{\infty}}{\oplus}$ & $\frac{\mathbb{8}}{\text { গே }}$ & 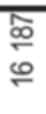 & 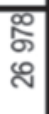 & 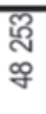 & $\underset{\sim}{\text { ন }}$ & $\begin{array}{l}8 \\
8 \\
ㅇ ㅡ\end{array}$ & $\begin{array}{l}8 \\
8 \\
8\end{array}$ & $\begin{array}{l}\text { ! } \\
\text { ల }\end{array}$ & $\begin{array}{l}\text { ले } \\
\sim\end{array}$ & $\begin{array}{l}8 \\
8 \\
\text { m }\end{array}$ & 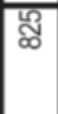 & 0 & 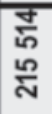 \\
\hline & $\begin{array}{l}\infty \\
\text { 定 }\end{array}$ & 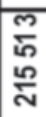 & $\begin{array}{l}\bar{\Phi} \\
\bar{\emptyset}\end{array}$ & 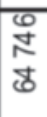 & 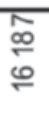 & $\frac{\tilde{\sigma}}{\stackrel{Ð}{g}}$ & $\underset{\infty}{\stackrel{\infty}{\sigma}}$ & 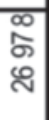 & $\begin{array}{l}\text { స్ } \\
\text { స్ } \\
\text { कo }\end{array}$ & ঙ্ণ & $\begin{array}{l}\text { 응 } \\
8 \\
\text { 으 }\end{array}$ & $\begin{array}{l}\text { 8) } \\
8 \\
\text { ㄱ. }\end{array}$ & $\begin{array}{l}\infty \\
\stackrel{\infty}{0} \\
\text { m }\end{array}$ & $\begin{array}{l}\text { ले } \\
\stackrel{N}{v}\end{array}$ & $\begin{array}{l}\text { O } \\
8 \\
\text { ल }\end{array}$ & జ్ల & 0 & 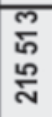 \\
\hline & 客 & $\begin{array}{l}\frac{N}{n} \\
\frac{N}{N}\end{array}$ & $\begin{array}{l}\bar{\Sigma} \\
\text { बे } \\
-\end{array}$ & $\begin{array}{l}\frac{G}{N} \\
\bar{\delta}\end{array}$ & $\stackrel{\grave{\infty}}{\oplus}$ & 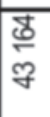 & $\stackrel{\grave{\infty}}{\oplus}$ & 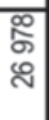 & $\begin{array}{l}\overline{\text { స్ }} \\
\text { o }\end{array}$ & ন্ণ & $\begin{array}{l}8 \\
8 \\
ㅇ\end{array}$ & $\begin{array}{l}8 \\
8 \\
\text { ㄴ. }\end{array}$ & $\frac{8}{6}$ & 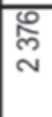 & $\begin{array}{l}\text { ষ্ } \\
\text { ল }\end{array}$ & జ్ల & 0 & $\begin{array}{l}\frac{\pi}{5} \\
\frac{\pi}{2} \\
\frac{\pi}{N}\end{array}$ \\
\hline & $\begin{array}{l}0 \\
\text { \&े }\end{array}$ & 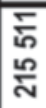 & $\begin{array}{l}\bar{\emptyset} \\
\bar{\emptyset}\end{array}$ & $\begin{array}{l}\frac{Q}{N} \\
\mathbb{J}\end{array}$ & $\stackrel{\frac{\pi}{c}}{\varphi}$ & 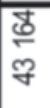 & $\stackrel{\infty}{\oplus}$ & 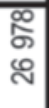 & 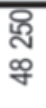 & $\underset{\sim}{\bar{N}}$ & $\begin{array}{l}8 \\
8 \\
ㅇ\end{array}$ & $\begin{array}{l}8 \\
8 \\
ㅇ\end{array}$ & $\frac{8}{8}$ & $\begin{array}{l}\text { लू } \\
\text { N }\end{array}$ & $\begin{array}{l} \\
\text { স্ল }\end{array}$ & 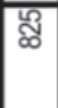 & 0 & 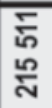 \\
\hline & م & 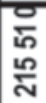 & $\begin{array}{l}\stackrel{2}{0} \\
\stackrel{0}{\circ}\end{array}$ & $\begin{array}{l}\text { N } \\
\mathbb{J}\end{array}$ & $\stackrel{\infty}{\oplus}$ & $\frac{\varrho}{\text { 乌ั }}$ & $\underset{\infty}{\infty}$ & 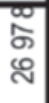 & $\begin{array}{l}\text { I } \\
\text { 孛 }\end{array}$ & ๙ & $\begin{array}{l}8 \\
8 \\
\text { 으 }\end{array}$ & 요 & $\stackrel{\infty}{\infty}$ & $\stackrel{\text { లे }}{\sim}$ & \& & జ & & 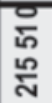 \\
\hline & ষ্ণ & $\begin{array}{l}\text { 읂 } \\
\text { 은 }\end{array}$ & $\begin{array}{l}\overline{2} \\
\text { ब్ }\end{array}$ & $\begin{array}{l}\frac{G}{N} \\
\bar{\Xi}\end{array}$ & $\stackrel{\infty}{\oplus}$ & $\frac{\mathbb{\Xi}}{\frac{\$}{g}}$ & $\stackrel{\Phi}{\oplus}$ & 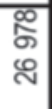 & $\begin{array}{l}\text { 㚣 } \\
\text { 字 }\end{array}$ & $\stackrel{\text { న్ }}{\text { న }}$ & $\begin{array}{l}8 \\
8 \\
ㅇ ㅡ\end{array}$ & 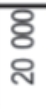 & $\frac{8}{\infty}$ & $\begin{array}{l}\text { ల్ } \\
\text { N }\end{array}$ & $\begin{array}{l}\text { ষ্ষ } \\
\text { ল }\end{array}$ & 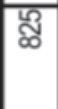 & 0 & $\begin{array}{l}\text { 은 } \\
\text { 온 } \\
\text { N }\end{array}$ \\
\hline & m & $\begin{array}{l}\text { 은 } \\
\text { 을 }\end{array}$ & 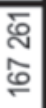 & $\begin{array}{l}\frac{Q}{N} \\
\text { J }\end{array}$ & $\stackrel{\infty}{\oplus}$ & $\frac{\mathbb{\Xi}}{\stackrel{Ð}{g}}$ & $\stackrel{\oplus}{\oplus}$ & $\begin{array}{l}\text { D } \\
\text { ద } \\
\stackrel{2}{N}\end{array}$ & 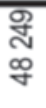 & న্ & $\begin{array}{l}8 \\
8 \\
ㅇ ㅡ\end{array}$ & 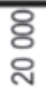 & $\frac{8}{\infty}$ & $\stackrel{\substack{\infty \\
\sim}}{\sim}$ & $\begin{array}{l}\text { প্ল } \\
\text { ল }\end{array}$ & స్ర & 0 & $\begin{array}{l}\text { 은 } \\
\text { 잇 }\end{array}$ \\
\hline & 尔 & 0 & 0 & & & & & & 0 & & & & & & & & 0 & 0 \\
\hline$\frac{8}{3}$ & 这 & 0 & 0 & & & & & & 0 & & & & & & & & 0 & 0 \\
\hline 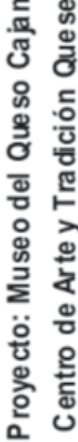 & 号 & 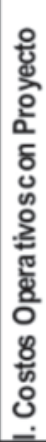 & 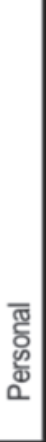 & 용 & 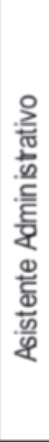 & 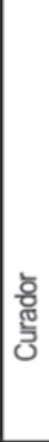 & 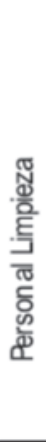 & 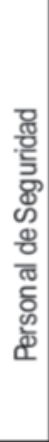 & 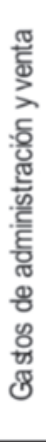 & 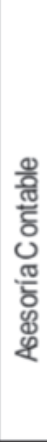 & 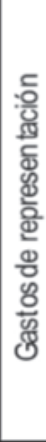 & 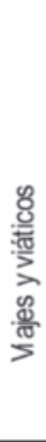 & 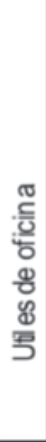 & 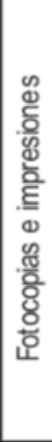 & 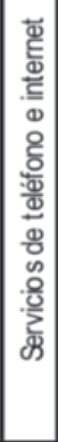 & 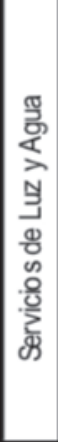 & 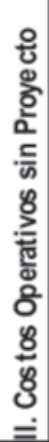 & 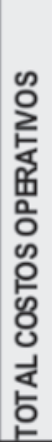 \\
\hline
\end{tabular}




\section{Hacia una nueva metodología de diseño de proyectos de inversión pública}

\section{Beneficios del proyecto}

La estimación de los beneficios del proyecto tiene por finalidad medir el grado de cambio económico que se puede atribuir a una intervención, eliminando el sesgo de factores ajenos al proyecto que podrían haber influido en los resultados obtenidos. Los beneficios obtenidos por el proyecto son de carácter incremental y atribuible sólo a la intervención. Los beneficios del proyecto permiten determinar con mayor certeza la relación causa-efecto entre un proyecto dado y sus efectos directos sobre la población beneficiaria. La metodología a seguir consiste en la construcción de un escenario contrafactual, a través del cual se comparan los ingresos en la situación con proyecto, versus los ingresos que se estima se obtendrían en una situación en la que el proyecto no fuera implementado. De este modo se puede estimar el incremento de los ingresos pueden ser efectivamente atribuidos al proyecto implementado.

El siguiente cuadro de beneficios muestra los ingresos incrementales generados por el proyecto del Museo del Queso en Cajamarca. Al término del mismo, se prevé que el impacto económico del proyecto será de S/.2'944,219.

\section{SUPUESTOS}

Con proyecto

Tasa de crec. de demanda de lácteos $\quad 5.00 \%$

Sin proyecto

Tasa de crec de demanda de lácteos $\quad 1.00 \%$ \begin{tabular}{ll} 
Tasa crec. demanda sector turismo & $5.00 \%$ \\
\hline
\end{tabular} Tasa crec. demanda sector turismo

\begin{tabular}{|c|c|c|c|c|c|c|c|c|c|c|c|c|}
\hline \multirow{2}{*}{ RUBROS } & \multirow{2}{*}{ ANTES } & \multicolumn{10}{|c|}{ DESPUES (INGRESOS ADICIONALES) } & \multirow{2}{*}{$\begin{array}{c}\text { SUYATORIA DE } \\
\text { NGRESOS } \\
\text { AOICIONALES } \\
\text { ANUALES }\end{array}$} \\
\hline & & 1 & 2 & 3 & 4 & 5 & 6 & 7 & 8 & 9 & 10 & \\
\hline Ingresos con Proyecto & 5853414 & 5853414 & 6086710 & 8280512 & 8656984 & 9050638 & 9462257 & 9892664 & 10342717 & 10813313 & 11305393 & 4291729 \\
\hline Ingresos Directos & 0 & 0 & 0 & 480635 & 501552 & 52342 & 546287 & 570195 & 595192 & 621329 & 648650 & \\
\hline Ingresos por boleteria* & 0 & 0 & 0 & 361361 & 377622 & 394615 & 412373 & 430930 & 450322 & 470580 & 491762 & \\
\hline Otros ingresos del museo"* & 0 & 0 & 0 & 119274 & 123929 & 128806 & 133914 & 139266 & 14487 & 150743 & 156894 & \\
\hline Ingresos indirectos & 5853414 & 5853414 & 6086710 & 7799877 & 8155433 & 8527217 & 8915970 & 9322469 & 974752 & 10191984 & 10656736 & \\
\hline Ingresos MYPES - lácteos*** & 860214 & 860214 & 868816 & 912257 & 957870 & 1005763 & 1056051 & 1108854 & 1164297 & 1222512 & 1283637 & \\
\hline Ingresos operadores turisticos ${ }^{* * * *}$ & 4993200 & 4993200 & 5217894 & 6887620 & 7197563 & 7521453 & 7859919 & 8213615 & 8583228 & 8969473 & 9373099 & \\
\hline Ingresos sin Proyecto & 5853414 & 5853414 & 6086710 & 6330204 & 6584350 & 6849626 & 7126529 & 7415580 & 771732 & 803232 & 8361174 & 23529917 \\
\hline Ingresos indirectos & 5853414 & 5853414 & 6086710 & 6330204 & 6584350 & 6849626 & 7126529 & 7415580 & 771732 & 803232 & 8361174 & \\
\hline Ingresos MYPES - lácteos & 860214 & 860214 & 868816 & 877504 & 886279 & 895142 & 904094 & 913134 & 922260 & 931488 & 940803 & \\
\hline Ingresos operadores turisticos & 4993200 & 4993200 & 5217894 & 5452699 & 5698071 & 5954484 & 6222436 & 6502445 & 6795055 & 7100833 & 7420370 & \\
\hline $\begin{array}{l}\text { NGRESOS INCREMENTALES (ATRIBUIBLES } \\
\text { AL PROYECTO) }\end{array}$ & & & 0 & 1950308 & 2072634 & 2201012 & 2335728 & 2477085 & 2625390 & 2780998 & 2944219 & \\
\hline
\end{tabular}

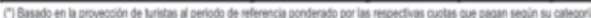

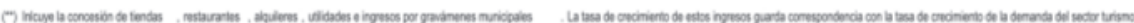

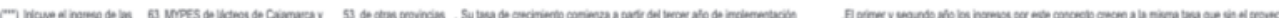




\section{David Medianero Burga}

\section{Evaluación del proyecto}

La evaluación del proyecto consiste en comparar los flujos de ingresos y egresos con la finalidad de estimar su rentabilidad privada y social, y sobre esta base decidir la conveniencia de aprobarlo e implementarlo. Es, en esencia, un análisis incremental, en el cual se compara la inversión del proyecto, con la diferencia resultante del incremento de los ingresos operativos menos el incremento de los costos operativos. El instrumento indispensable para la evaluación de un proyecto es el flujo de caja, el cual sintetiza todos los movimientos de efectivo que tendrán lugar durante la vida del proyecto, incluyendo tanto la etapa de ejecución como la etapa de operación. Cabe resaltar que el flujo de caja se construye para toda la vida del proyecto, periodo denominado también "horizonte de evaluación", a diferencia del presupuesto del proyecto, que sólo comprende la etapa de inversión, conocida como "horizonte de ejecución" del proyecto.

El flujo de caja es un instrumento que permite visualizar los resultados monetarios generados efectivamente en cada período durante todo el proceso que dure el proyecto. Es de mucha utilidad para realizar la evaluación económica de los proyectos al proporcionar indicadores que permiten decidir acerca de la aprobación o no de determinado proyecto. Se construye sobre la información que proporcionan el plan operativo global, los costos operativos incrementales y la evaluación de impacto.

En el siguiente ejemplo del Proyecto de Museo del Queso en Cajamarca, una vez evaluado el flujo de caja del proyecto, los indicadores de rentabilidad obtenidos señalan un VAN privado de S/. 54,531 y una TIR privada de $12.76 \%$. Por otro lado, al considerar los beneficios indirectos del proyecto, los indicadores sociales obtenidos arrojan un VAN social de S/. 7'158,014 con una TIR social mucho mayor, de 59.10\%. La tasa de descuento es del $12 \%$. 


\begin{tabular}{|c|c|c|c|c|c|c|c|c|c|c|c|}
\hline PARTDAS & Año 0 & Anto 1 & An̂००2 & Año3 & Año 4 & Año 5 & คก้อ 6 & Año 7 & Afro 8 & Año 9 & Año 10 \\
\hline I. MOD ULO DE INVERS ON & 1257853 & & & & & & & & & & \\
\hline L Diseño y co nstrucoö nde la in fra estructua & 545000 & & & & & & & & & & \\
\hline 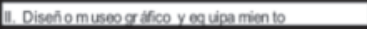 & 47958 & & & & & & & & & & \\
\hline \multicolumn{12}{|l|}{ 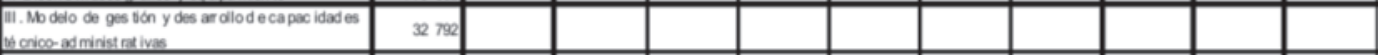 } \\
\hline \multicolumn{12}{|l|}{ N. I mpie me riación de e stra tegias de pu blicidad. } \\
\hline V. Se ha gesfo nod o e ficazm en te el pro yocto. & 156049 & & & & & & & & & & \\
\hline 1. MODULO DE OPERACION & 0 & & & 1734792 & 1857125 & 1985502 & 2120218 & 2261573 & 2409883 & 2565472 & 2728704 \\
\hline 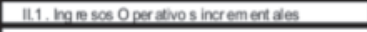 & & & & 1950308 & 2072634 & 2201012 & 233572 & 2477005 & 2625396 & 278099 & 2944219 \\
\hline A. ingresosepenafives con po verto & & 5853414 & 6006710 & 8280512 & 8656984 & 9050630 & 946225 & 9892604 & 10342711 & 10813313 & 11305,393 \\
\hline ngasesos D rectos & & & & 480635 & 501552 & 523421 & 54628 & 570,196 & 59519 & 2.1390 & 648656 \\
\hline Ingeses indisclos & & 5853.414 & 6006710 & 770987 & 8155433 & 8527217 & 8915970 & 932240 & 9747524 & $10.1919 \mathrm{ges}$ & 10656736 \\
\hline B. Ingresos coesa fivos sinprovecto & & 5.853 .414 & 6000710 & 6330204 & 6584350 & 6849626 & 7126529 & $7415 \mathrm{sed}$ & 771732 & 8002331 & 8351.174 \\
\hline Inge sos indis clos & & 5853.414 & 6006710 & 6330204 & 6584350 & 6849626 & 7126529 & $7415 \mathrm{sed}$ & 7717,321 & 8092321 & 8351174 \\
\hline 1112.Gasios Coerativas incrementies & & & & 215.510 & 215510 & 215510 & 215511 & 215512 & 215513 & 215514 & 215515 \\
\hline A. Gast cs oper filivo s con provecto & & & & 215510 & 21.5510 & 215510 & 215511 & 215.512 & 215.513 & 215514 & 215.515 \\
\hline \multicolumn{12}{|l|}{ B. Gast os oper afivo s sin pro yecto } \\
\hline \multicolumn{12}{|l|}{ II.EVALUACION PRNADA } \\
\hline UL.1Flue decaian eto rivado mominal & -1257.853 & & & 265.126 & 286042 & 307912 & $3307 \pi$ & 35468 & $379 \mathrm{egd}$ & 405815 & 433142 \\
\hline II Fluio decaian eto rivado actualizado & -1257853 & & & 18871 & 18178 & 174717 & 16758 & 160441 & 153348 & 146331 & 139460 \\
\hline \multicolumn{12}{|l|}{ U1.3indicadores de Rertabilidad Pivada } \\
\hline VAN privado & 54531 & & & & & & & & & & \\
\hline TiR privada & $1276 \%$ & & & & & & & & & & \\
\hline \multicolumn{12}{|l|}{ N.EVAW ACION SOCIAL } \\
\hline N.1 Aujode caia ne bso cial nominal & -1257853 & & & 173479 & 1857125 & 1985509 & 2120218 & 2261578 & 240988 & 2565.479 & 2728704 \\
\hline 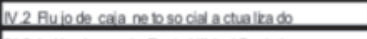 & -125785 & & & 1234796 & 1180236 & 1126627 & 107416 & 1023021 & 973311 & 925.139 & 878.57 \\
\hline \multicolumn{12}{|l|}{ N. 3 ind te adoms on Rent ablilidad Social } \\
\hline VAN social & 7158014 & & & & & & & & & & \\
\hline TIR socia & $5910 \%$ & & & & & & & & & & \\
\hline
\end{tabular}

\section{Monitoreo y evaluación}

Con la finalidad de realizar una adecuada labor de monitoreo y evaluación del proyecto durante su ejecución y a su conclusión, el estudio de preinversión deberá establecer un conjunto de indicadores que permiten medir el desempeño de la intervención, en términos de sus logros, tanto de aquellos de carácter interno como de aquellos relacionados al bienestar de la población beneficiaria. La matriz de monitoreo y evaluación es un instrumento que permite realizar un seguimiento del desempeño del proyecto en el transcurso del tiempo, permitiendo verificar si los objetivos planteados inicialmente están siendo alcanzados.

Cabe destacar dos hechos importantes. Por un lado, el hecho de que la matriz de monitoreo y evaluación constituye una síntesis de todos los estudios realizados, en lo que se refiere a sus expresiones cuantitativas; y por otro lado, el hecho de que la base de este instrumentos se encuentra en el marco lógico del proyecto. 


\section{David Medianero Burga}

En términos resumidos, en el siguiente ejemplo, mediante el uso de la Matriz de Monitoreo y Evaluación, el proyecto busca incrementar a 43800 el número de visitantes anuales y 2.4 días el tiempo de permanencia en el lugar.

Proyecto: Museo del Queso Cajamarquino,

Formato: Matriz de

Centro de Arte y Tradición Quesera monitoreo y evaluación

\begin{tabular}{|c|c|c|c|c|c|c|}
\hline \multicolumn{4}{|c|}{ INDICADORES } & \multirow{2}{*}{ LINEA DE BASE } & \multirow{2}{*}{ HITOS } & \multirow{2}{*}{ METAS } \\
\hline TIPO & OBJETINO & NOMBRE PROPUESTO & DEFINICION OPERATIVA & & & \\
\hline \multirow{3}{*}{ IMPACTO } & \multirow{3}{*}{ 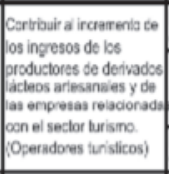 } & $\begin{array}{l}\text { agresas po ventas de las W/YPES } \\
\text { de lactocs. }\end{array}$ & Se refiere a los irgrescs por la verita de productos lácteos. & 4000 soles mensuales. & \begin{tabular}{|l|}
4200 soles mansuales a \\
fines dei primer alio
\end{tabular} & 4400 soles mensuales \\
\hline & & $\begin{array}{l}\text { Ingresos por ventas de los } \\
\text { operadores turisticos. }\end{array}$ & $\begin{array}{l}\text { Se refiere a los ingrescos por la venta ce sanicios de los } \\
\text { cperadores turistocs (agencias de viąe, restaurantes. } \\
\text { nospedajes, etc.) }\end{array}$ & $\begin{array}{l}\text { g. dolares generados por el } \\
\text { fiempo peomedig de } \\
\text { permanenocia del turista en } \\
\text { Cajamarca. }\end{array}$ & \begin{tabular}{|l|}
200 tdaras a limino dal \\
griner anho
\end{tabular} & 239 doblares \\
\hline & & $\begin{array}{l}\text { Nùmero de empleos directos } \\
\text { generados }\end{array}$ & $\begin{array}{l}\text { Se refere al nùmero de empleos generados como } \\
\text { consecuencia directa del proyocto. }\end{array}$ & 0 & Por det & P.D \\
\hline \multirow{3}{*}{ EFECTO } & \multirow{3}{*}{$\begin{array}{l}\text { Implementar y hacer } \\
\text { tuncionar ol museo dol } \\
\text { queso Caganarzuino cone } \\
\text { una nueva actividad } \\
\text { turistica en el Ciroulto } \\
\text { Regional Norie. }\end{array}$} & Nünevo de visitanites del museo. & Se refiere al rùmero de personas que visitan el museo. & 0 & \begin{tabular}{|l|}
$2000 t$ vilurtos à primar \\
ahio
\end{tabular} & 43800 anuales. \\
\hline & & 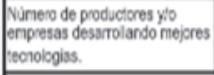 & $\begin{array}{l}\text { Se refere a la cantidad de perscenas que usan nuevas } \\
\text { tecnologlas y.o métcodos de gestión emprosarial como } \\
\text { tesultado de la capacitación. }\end{array}$ & P.D $(*)$ & $\begin{array}{l}40 \text { produclanos a terrieo } \\
\text { del priner anio }\end{array}$ & 63 productores \\
\hline & & $\begin{array}{l}\text { Tienco promedio de permarencia } \\
\text { del tursta on Cajanarca. }\end{array}$ & $\begin{array}{l}\text { Se vefiere al temso prorredio de perrarercia del luista en } \\
\text { Cajamarca como corsocuoncia do incromento do la dorta } \\
\text { uvistica. }\end{array}$ & 1.9 dias & Por det & 2.4 dias \\
\hline \multirow{7}{*}{ PROOUCTO } & \begin{tabular}{|l|} 
Diseho y constowodin do \\
a infraestuctura.
\end{tabular} & Area construida. & 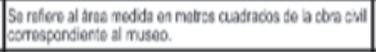 & 0 & \begin{tabular}{|l|}
500 retros cuadrados al \\
temino del pinter aflo
\end{tabular} & Tro nestos cuscresos \\
\hline & \multirow{2}{*}{$\begin{array}{l}\text { 1. Diseño nuseográfico y } \\
\text { equipariento }\end{array}$} & Número de colecciones. & 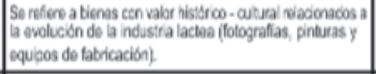 & 0 & Q ipos de coieccenes a & Dof tipns de eniercienes \\
\hline & & Número de equipos & $\begin{array}{l}\text { Se refere a bs equipos, herrarierias, utensilos y muetles } \\
\text { relacionados al proceso de producción da la incustra lictea. }\end{array}$ & 0 & $\begin{array}{l}\text { 4. equipos al terriro to } \\
\text { priner ańo }\end{array}$ & 60 equipos \\
\hline & \multirow{2}{*}{$\begin{array}{l}\text { II. Modelo de gestion y } \\
\text { dasamalo de capaciades } \\
\text { tecrico-administrativas }\end{array}$} & Museo constituido legalrrente & $\begin{array}{l}\text { Se refiere a la constitución legal y a los irstrumentos de } \\
\text { gostion. }\end{array}$ & 0 & Por det & 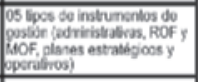 \\
\hline & & $\begin{array}{l}\text { Nimneo de trabajadoness } \\
\text { oapeotados }\end{array}$ & $\begin{array}{l}\text { Se refien al persona do planta del ruseo capacitado en las } \\
\text { tareas funcicnaies del musco. }\end{array}$ & 0 & $\begin{array}{l}\text { 3 trabaidores al termino } \\
\text { del pintor alio }\end{array}$ & 12 trabajadores \\
\hline & \begin{tabular}{|l|} 
V. Implementación de \\
estralepas de publicidad.
\end{tabular} & $\begin{array}{l}\text { Nürneco de oceradores turistocs } \\
\text { que ncorporan al museo corro } \\
\text { parte de su clerta turistica. } \\
\end{array}$ & $\begin{array}{l}\text { Se refere a las agencia de viaje, hoteles, empresas de } \\
\text { transportes que atenden a buristas que vistan el museo. }\end{array}$ & 0 & $\begin{array}{l}\text { 30 operadcres turisficts } \\
\text { al ternino dal priner afo }\end{array}$ & 63 operadores \\
\hline & $\begin{array}{l}\text { Y. Se ha gestionado } \\
\text { eficazmerte el proyecto. }\end{array}$ & $\begin{array}{l}\text { Nurrere ce nótres da róntoneoy } \\
\text { avaluación. }\end{array}$ & $\begin{array}{l}\text { Se refiere a los intorres de rnonitcres tinestral y los nfornes } \\
\text { de evaluacićn de medio térnino y final. }\end{array}$ & 0 & $\begin{array}{l}4 \text { infomes da menitoras } \\
\text { al tierrino dal frimer afio }\end{array}$ & 10 \\
\hline
\end{tabular}

\section{Notas Referenciales}

1 VAN : Valor Actual Neto 
Hacia una nueva metodologia de diseño de proyectos de inversión pública

\section{Bibliografía}

Agencia Española de Cooperación Internacional (AECI); Ministerio de Asuntos Exteriores, Metodología de Gestión de Proyectos de la Cooperación Española, Madrid: Agencia Española de Cooperación Internacional, 2000.

Asociación Los Andes de Cajamarca, Promoción del Desarrollo Humano Sostenible, Glosario de Términos. Cajamarca: ALAC, 2007.

Belli, Pedro; Anderson, Jock; Bamum, Howard; Dixon, John; Tan, Jec-peng, Handbook on Economic Analysis of Investment Operations, Operation Policy Department Learning and Leadership Center.

Comisión Europea, Manual de Gestión del Ciclo de Proyecto, Unidad de Evaluación de la Oficina de Cooperación EuropeAid, 2001.

Contreras, Eduardo, Evaluación social de inversiones públicas: enfoques alternativos y su aplicabilidad para Latinoamérica, Serie Manuales No 37, Santiago de Chile: Instituto Latinoamericano dePlanificación Económica y Social (ILPES), 2004.

Departamento Nacional de Planeación, Manual Metodológico General para la Identificación, Preparación y Evaluación de Proyectos, Colombia: Banco de Programas y Proyectos de Inversión Nacional, 2003.

Departamento Nacional de Planeación. Guía de Gestión de Proyectos para la Comunidad. Bogotá: Banco Nacional de Programas y Proyectos de Inversión, 2003.

European Commission. Project Cycle Management Guidelines - Volumen 1, Brussels: Office for Official Publications of the European Communities, 2004.

enkins, Glenn P. Harberger Arnold c. Manual Análisis de Costo Beneficio de las Decisiones de Inversión 2da. Edición, Estados Unidos, Harvard Institute for Internacional Development, 2005.

Medianero, David, El Enfoque del Marco Lógico, Lima: Fondo Nacional de Capacitación Laboral y Promoción del Empleo (FONDOEMPLEO), 2007.

Medianero, David, Metodología de Diseño de Proyectos, Lima: Fondo Nacional de Capacitación Laboral y Promoción del Empleo (FONDOEMPLEO), 2007.

Ministerio de Economía y Finanzas. Guía General de Identificación, Formulación y Evaluación Social de Proyectos de Inversión Pública a nivel de Perfil, Lima: Dirección General de Programación Multianual, 2003. 